DESY 04-193

\title{
Single-spin asymmetries: the Trento conventions
}

\author{
Alessandro Bacchetta* \\ Institut für Theoretische Physik, Universität Regensburg, D-93040 Regensburg, Germany \\ Umberto D'Alesid $\dagger$ \\ INFN, Sezione di Cagliari and Dipartimento di Fisica, \\ Università di Cagliari, I-09042 Monserrato, Italy \\ Markus Dieh $¥$ \\ Deutsches Elektronen-Synchroton DESY, D-22603 Hamburg, Germany \\ C. Andy Millen $\$$ \\ TRIUMF, Vancouver, British Columbia V6T 2A3, Canada
}

\begin{abstract}
During the workshop "Transversity: New Developments in Nucleon Spin Structure" (ECT*, Trento, Italy, 14-18 June 2004), a series of recommendations was put forward by the participants concerning definitions and notations for describing effects of intrinsic transverse momentum of partons in semi-inclusive deep inelastic scattering.
\end{abstract}

\section{DEFINITION OF TRANSVERSE-MOMENTUM DEPENDENT FUNCTIONS}

A standard set of definitions and notations for transverse-momentum dependent distribution and fragmentation functions is given in Refs. 1, 2, 3]. We note that the definition of the antisymmetric tensor in those articles and in the present note is such that

$$
\epsilon^{0123}=+1
$$

Transverse-momentum dependent parton distributions of leading twist can be interpreted as number densities (see e.g. Refs. [4, 5, 6]). To connect with this interpretation, we take the example of the distribution of unpolarized quarks in a polarized proton, which is given $b^{1}$

$$
\begin{aligned}
f_{q / p^{\uparrow}}\left(x, k_{T}\right) & =f_{1}^{q}\left(x, k_{T}^{2}\right)-f_{1 T}^{\perp q}\left(x, k_{T}^{2}\right) \frac{\epsilon^{\mu \nu \rho \sigma} P_{\mu} k_{\nu} S_{\rho} n_{\sigma}}{M(P \cdot n)} \\
& =f_{1}^{q}\left(x, k_{T}^{2}\right)-f_{1 T}^{\perp q}\left(x, k_{T}^{2}\right) \frac{\left(\hat{\boldsymbol{P}} \times \boldsymbol{k}_{T}\right) \cdot \boldsymbol{S}}{M}
\end{aligned}
$$

where $f_{1}^{q}$ is the unpolarized quark density and $f_{1 T}^{\perp q}$ describes the Sivers effect [7]. Here $P$ is the momentum of the proton, $S$ is its covariant spin vector normalized to $S^{2}=-1$, and $M$ is the proton mass. The covariant definition of parton distributions requires an auxiliary lightlike vector $n$, which plays the role of a preferred direction in a given physical process. ${ }^{2}$ Furthermore, $k$ is the momentum of the quark, $k_{T}$ its component perpendicular to $P$ and $n$, and $x=(k \cdot n) /(P \cdot n)$ its light-cone momentum fraction. The second expression in (2) holds in any frame where $\boldsymbol{n}$ and the direction $\hat{\boldsymbol{P}}$ of the proton momentum point in opposite directions. ${ }^{3}$ Therefore $f_{1 T}^{\perp q}>0$ corresponds to a preference of

\footnotetext{
*Electronic address: alessandro.bacchetta@physik.uni-r.de

${ }^{\dagger}$ Electronic address: umberto.dalesio@ca.infn.it

$\ddagger$ Electronic address: markus.diehl@desy.de

$\S$ Electronic address: miller@triumf.ca

${ }^{1}$ The following expression is obtained from the quark correlation function in Eq. (2) of Ref. [2] by identifying $n=n_{-}$, multiplying with $\not h / 2$ and taking the trace.

2 This direction can for instance be taken along the virtual photon momentum in deep inelastic scattering, or along the momentum of the second incoming hadron in Drell-Yan lepton pair production. Other choices of $n$ are possible, provided that the corresponding changes in the result are sufficiently suppressed by inverse powers of the large momentum scale.

${ }^{3}$ We use the four-vector $k_{T}$ and its square as arguments in the distribution functions to emphasize that they are Lorentz invariant. One may instead use $\boldsymbol{k}_{T}$ if it is clear from the context to which frame the vectors refer.
} 
the quark to move to the left if the proton is moving towards the observer and the proton spin is pointing upwards. In the convention of Ref. [8] the Sivers effect is described by

$$
f_{q / p^{\uparrow}}\left(x, k_{T}\right)-f_{q / p^{\uparrow}}\left(x,-k_{T}\right)=\Delta^{N} f_{q / p^{\uparrow}}\left(x, k_{T}^{2}\right) \frac{\left(\hat{\boldsymbol{P}} \times \boldsymbol{k}_{T}\right) \cdot \boldsymbol{S}}{\left|\boldsymbol{k}_{T}\right|}
$$

so that

$$
\Delta^{N} f_{q / p^{\uparrow}}\left(x, k_{T}^{2}\right)=-\frac{2\left|\boldsymbol{k}_{T}\right|}{M} f_{1 T}^{\perp q}\left(x, k_{T}^{2}\right) .
$$

Either $f_{1 T}^{\perp q}$ or $\Delta^{N} f_{q / p^{\uparrow}}$ may be referred to as the "Sivers function". It is strongly encouraged that authors use one or the other of these notations, or provide the relation of the functions they might use to the ones discussed here.

Let us give the corresponding relation for the Boer-Mulders function, introduced in Ref. 2]. The distribution of transversely polarized quarks in an unpolarized proton is ${ }^{4}$

$$
\begin{aligned}
f_{q^{\uparrow} / p}\left(x, k_{T}\right) & =\frac{1}{2}\left(f_{1}^{q}\left(x, k_{T}^{2}\right)-h_{1}^{\perp q}\left(x, k_{T}^{2}\right) \frac{\epsilon^{\mu \nu \rho \sigma} P_{\mu} k_{\nu} S_{q \rho} n_{\sigma}}{M(P \cdot n)}\right) \\
& =\frac{1}{2}\left(f_{1}^{q}\left(x, k_{T}^{2}\right)-h_{1}^{\perp q}\left(x, k_{T}^{2}\right) \frac{\left(\hat{\boldsymbol{P}} \times \boldsymbol{k}_{T}\right) \cdot \boldsymbol{S}_{q}}{M}\right),
\end{aligned}
$$

where $S_{q}$ is the covariant spin vector of the quark. Introducing

$$
f_{q^{\uparrow} / p}\left(x, k_{T}\right)-f_{q^{\uparrow} / p}\left(x,-k_{T}\right)=\Delta^{N} f_{q^{\uparrow} / p}\left(x, k_{T}^{2}\right) \frac{\left(\hat{\boldsymbol{P}} \times \boldsymbol{k}_{T}\right) \cdot \boldsymbol{S}_{q}}{\left|\boldsymbol{k}_{T}\right|}
$$

we get the relation

$$
\Delta^{N} f_{q^{\uparrow} / p}\left(x, k_{T}^{2}\right)=-\frac{\left|\boldsymbol{k}_{T}\right|}{M} h_{1}^{\perp q}\left(x, k_{T}^{2}\right) .
$$

Likewise there are two common notations for the Collins fragmentation function [10]. With the conventions of Refs. [1, 2, 3] the number density of an unpolarized hadron $h$ in a transversely polarized quark is 5

$$
\begin{aligned}
D_{h / q^{\uparrow}}\left(z, P_{h T}\right) & =D_{1}^{q}\left(z, P_{h T}^{2}\right)-H_{1}^{\perp q}\left(z, P_{h T}^{2}\right) \frac{\epsilon^{\mu \nu \rho \sigma} P_{h \mu} k_{\nu} S_{q \rho} n_{\sigma}^{\prime}}{M_{h}\left(P_{h} \cdot n^{\prime}\right)} \\
& =D_{1}^{q}\left(z, P_{h T}^{2}\right)+H_{1}^{\perp q}\left(z, P_{h T}^{2}\right) \frac{\left(\hat{\boldsymbol{k}} \times \boldsymbol{P}_{h T}\right) \cdot \boldsymbol{S}_{q}}{z M_{h}}
\end{aligned}
$$

where the measure of the density is $d z d^{2} P_{h T}$. Here $D_{1}^{q}$ is the unpolarized fragmentation function, $P_{h}$ is the hadron momentum, $M_{h}$ its mass, $k$ is the momentum of the quark, $S_{q}$ its covariant spin vector, and $n^{\prime}$ an auxiliary lightlike vector. Furthermore, $z=\left(P_{h} \cdot n^{\prime}\right) /\left(k \cdot n^{\prime}\right)$ is the light-cone momentum fraction of the hadron with respect to the fragmenting quark, and $P_{h T}$ the component of $P_{h}$ transverse to $k$ and $n^{\prime}$. One can trade $P_{h T}$ for $k_{T}=-P_{h T} / z$, the component of $k$ transverse to $P_{h}$ and $n^{\prime}$. The second line of (8) holds in frames where $\boldsymbol{n}^{\prime}$ and the direction $\hat{\boldsymbol{k}}$ of the quark momentum point in opposite directions. Therefore, $H_{1}^{\perp q}>0$ corresponds to a preference of the hadron to move to the left if the quark is moving away from the observer and the quark spin is pointing upwards. In the notation of [1] the Collins effect is described by

$$
D_{h / q^{\uparrow}}\left(z, P_{h T}\right)-D_{h / q^{\uparrow}}\left(z,-P_{h T}\right)=\Delta^{N} D_{h / q^{\uparrow}}\left(z, P_{h T}^{2}\right) \frac{\left(\hat{\boldsymbol{k}} \times \boldsymbol{P}_{h T}\right) \cdot \boldsymbol{S}_{q}}{\left|\boldsymbol{P}_{h T}\right|}
$$

\footnotetext{
4 The following expression is obtained by identifying $n=n_{-}$, setting $S_{T}$ and $\lambda$ to zero, multiplying Eq. (2) in Ref. [2] with $\gamma^{\mu} n_{\mu} / 2+$ $i \sigma_{\mu \nu} \gamma_{5} n^{\mu} S_{q}^{\nu} / 2$, taking the trace and dividing by 2. See Eq. (11) and (12) of 9 for this connection to the number density interpretation.

${ }^{5}$ The following expression is obtained by identifying $n^{\prime}=n_{+}$, setting $S_{h T}$ and $\lambda_{h}$ to zero, multiplying Eq. (5) in Ref. [2] with $\gamma^{\mu} n_{\mu}^{\prime} / 2+$ $i \sigma_{\mu \nu} \gamma_{5} n^{\prime \mu} S_{q}^{\nu} / 2$ and taking the trace. See Eqs. (40) and (41) of [9].
} 
so that

$$
\Delta^{N} D_{h / q^{\uparrow}}\left(z, P_{h T}^{2}\right)=\frac{2\left|\boldsymbol{P}_{h T}\right|}{z M_{h}} H_{1}^{\perp q}\left(z, P_{h T}^{2}\right)
$$

Either $H_{1}^{\perp q}$ or $\Delta^{N} D_{h / q^{\uparrow}}$ may be referred to as "Collins function". Our relations (4), (7), (10) agree with (4.8.3a), (4.8.3b), (6.5.11) in Ref. [6].

We finally discuss the analog of the Sivers function in fragmentation, introduced by Mulders and Tangerman in Ref. [1]. The number density of a polarized spin-half hadron $h$ in an unpolarized quark is ${ }^{6}$

$$
\begin{aligned}
D_{h^{\uparrow} / q}\left(z, P_{h T}\right) & =\frac{1}{2}\left(D_{1}^{q}\left(z, P_{h T}^{2}\right)-D_{1 T}^{\perp q}\left(z, P_{h T}^{2}\right) \frac{\epsilon^{\mu \nu \rho \sigma} P_{h \mu} k_{\nu} S_{h \rho} n_{\sigma}^{\prime}}{M_{h}\left(P_{h} \cdot n^{\prime}\right)}\right) \\
& =\frac{1}{2}\left(D_{1}^{q}\left(z, P_{h T}^{2}\right)+D_{1 T}^{\perp q}\left(z, P_{h T}^{2}\right) \frac{\left(\hat{\boldsymbol{k}} \times \boldsymbol{P}_{h T}\right) \cdot \boldsymbol{S}_{h}}{z M_{h}}\right),
\end{aligned}
$$

where $S_{h}$ is the covariant spin vector of the hadron. As indicated in Ref. [12], we can write

$$
D_{h^{\uparrow} / q}\left(z, P_{h T}\right)-D_{h^{\uparrow} / q}\left(z,-P_{h T}\right)=\Delta^{N} D_{h \uparrow / q}\left(z, P_{h T}^{2}\right) \frac{\left(\hat{\boldsymbol{k}} \times \boldsymbol{P}_{h T}\right) \cdot \boldsymbol{S}_{h}}{\left|\boldsymbol{P}_{h T}\right|},
$$

which leads to ${ }^{7}$

$$
\Delta^{N} D_{h \uparrow / q}\left(z, P_{h T}^{2}\right)=\frac{\left|\boldsymbol{P}_{h T}\right|}{z M_{h}} D_{1 T}^{\perp q}\left(z, P_{h T}^{2}\right) .
$$

The definition of each parton distribution contains a Wilson line, which describes interactions with the spectator partons before or after the hard-scattering process. The path of this Wilson line in space-time is selected by the hard process in which the parton distribution appears. Each such path corresponds to its own set of distribution functions, which thus give the number of quarks found in the presence of the specified spectator interactions. Different paths can lead to different distributions, and the path should be specified in the notation when it is not evident from the context. $^{8}$ Using time reversal symmetry one can show 13

$$
f_{1}^{\mathrm{DIS}}\left(x, k_{T}^{2}\right)=f_{1}^{\mathrm{DY}}\left(x, k_{T}^{2}\right), \quad f_{1 T}^{\perp \mathrm{DIS}}\left(x, k_{T}^{2}\right)=-f_{1 T}^{\perp \mathrm{DY}}\left(x, k_{T}^{2}\right),
$$

where the superscripts respectively specify the distributions with Wilson lines appropriate for semi-inclusive deep inelastic scattering (SIDIS) and for Drell-Yan lepton pair production.

Wilson lines with a path selected by the process also appear in the definition of fragmentation functions. The relation between the functions relevant for different processes (such as $e^{+} e^{-}$annihilation or SIDIS) is currently under study.

\section{AZIMUTHAL ANGLES IN SEMI-INCLUSIVE DEEP INELASTIC SCATTERING}

A recommendation is made concerning the azimuthal angles relevant in the semi-inclusive cross section for

$$
\ell(l)+p(P) \rightarrow \ell\left(l^{\prime}\right)+h\left(P_{h}\right)+X
$$

where $\ell$ denotes the beam lepton, $p$ the proton target, and $h$ the produced hadron. As usual we define $q=l-l^{\prime}$ and $Q^{2}=-q^{2}$. The azimuthal angle $\phi_{h}$ between the lepton and the hadron planes should be defined as

$$
\begin{aligned}
\cos \phi_{h} & =\frac{(\hat{\boldsymbol{q}} \times \boldsymbol{l})}{|\hat{\boldsymbol{q}} \times \boldsymbol{l}|} \cdot \frac{\left(\hat{\boldsymbol{q}} \times \boldsymbol{P}_{h}\right)}{\left|\hat{\boldsymbol{q}} \times \boldsymbol{P}_{h}\right|}, \\
\sin \phi_{h} & =\frac{\left(\boldsymbol{l} \times \boldsymbol{P}_{h}\right) \cdot \hat{\boldsymbol{q}}}{|\hat{\boldsymbol{q}} \times \boldsymbol{l}|\left|\hat{\boldsymbol{q}} \times \boldsymbol{P}_{h}\right|},
\end{aligned}
$$

\footnotetext{
6 The following expression is obtained by identifying $n^{\prime}=n_{+}$, multiplying Eq. (5) in Ref. [2] with $\not h^{\prime} / 2$, taking the trace and dividing by 2 .

7 Note that there is a factor -2 too much in Eq. (5) of Ref. [12]. This does not affect any results in that work.

8 This has been realized only recently, and the necessary distinction is not made in [1, 2, 3, 4, 5, 6, 7, 8, 9, 10, 11, 12.
} 
with $\hat{\boldsymbol{q}}=\boldsymbol{q} /|\boldsymbol{q}|$, where all vectors refer to the target rest frame (or to any frame reached from the target rest frame by a boost along $\hat{\boldsymbol{q}}$ ). Writing the right-hand sides of (16) in a Lorentz invariant form, one has

$$
\begin{aligned}
\cos \phi_{h} & =-\frac{g_{\perp}^{\mu \nu} l_{\mu} P_{h \nu}}{\left|l_{\perp}\right|\left|P_{h \perp}\right|}, \\
\sin \phi_{h} & =-\frac{\epsilon_{\perp}^{\mu \nu} l_{\mu} P_{h \nu}}{\left|l_{\perp}\right|\left|P_{h \perp}\right|}
\end{aligned}
$$

with $\left|l_{\perp}\right|=\sqrt{-g_{\perp}^{\mu \nu} l_{\mu} l_{\nu}}$ and $\left|P_{h \perp}\right|=\sqrt{-g_{\perp}^{\mu \nu} P_{h \mu} P_{h \nu}}$. Here we introduced perpendicular projection tensors

$$
\begin{aligned}
& g_{\perp}^{\mu \nu}=g^{\mu \nu}-\frac{q^{\mu} P^{\nu}+P^{\mu} q^{\nu}}{P \cdot q\left(1+\gamma^{2}\right)}+\frac{\gamma^{2}}{1+\gamma^{2}}\left(\frac{q^{\mu} q^{\nu}}{Q^{2}}-\frac{P^{\mu} P^{\nu}}{M^{2}}\right), \\
& \epsilon_{\perp}^{\rho \sigma}=\epsilon^{\mu \nu \rho \sigma} \frac{P_{\mu} q_{\nu}}{P \cdot q \sqrt{1+\gamma^{2}}}
\end{aligned}
$$

with $\gamma=2 x M / Q$, where $x$ is the Bjorken variable and $M$ again the target mass. Evaluating the right-hand sides of (17) in the target rest frame, one recovers (16). The azimuthal angle $\phi_{S}$ relevant for specifying the target polarization is defined in analogy to (16) and (17), with $P_{h}$ replaced by the covariant spin vector $S$ of the target. The definitions of $\phi_{h}$ and $\phi_{S}$ are illustrated in Fig. 11. We emphasize that (16), (17), (18) do not depend on the choice of coordinate axes. For definiteness we show in Fig. 1 one frequently used coordinate system. In this system the tensors defined in Eq. (18) have nonzero components $g_{\perp}^{11}=g_{\perp}^{22}=-1$ and $\epsilon_{\perp}^{12}=-\epsilon_{\perp}^{21}=-1$. Note that two different conventions for drawing angles and interpreting their sign in figures are in general use in the literature:

A. The $z$ axis is specified and angles are drawn as arcs with one arrowhead. If an angle is oriented according to the right-hand rule it is positive, otherwise it is negative. Fig. 11 illustrates the application of this convention.

B. Illustrated angles are always assumed to be positive. Only the location of the arc affects the definition of the angle. No orientation should be assigned to the arc, and any $z$ axis that may be present does not affect the angle definition.

It is strongly recommended that authors avoid placing single arrowheads on arcs when using convention $\mathrm{B}$. When using convention A an explicit remark in the caption may be useful when the figure illustrates a situation in which an angle has a negative value.

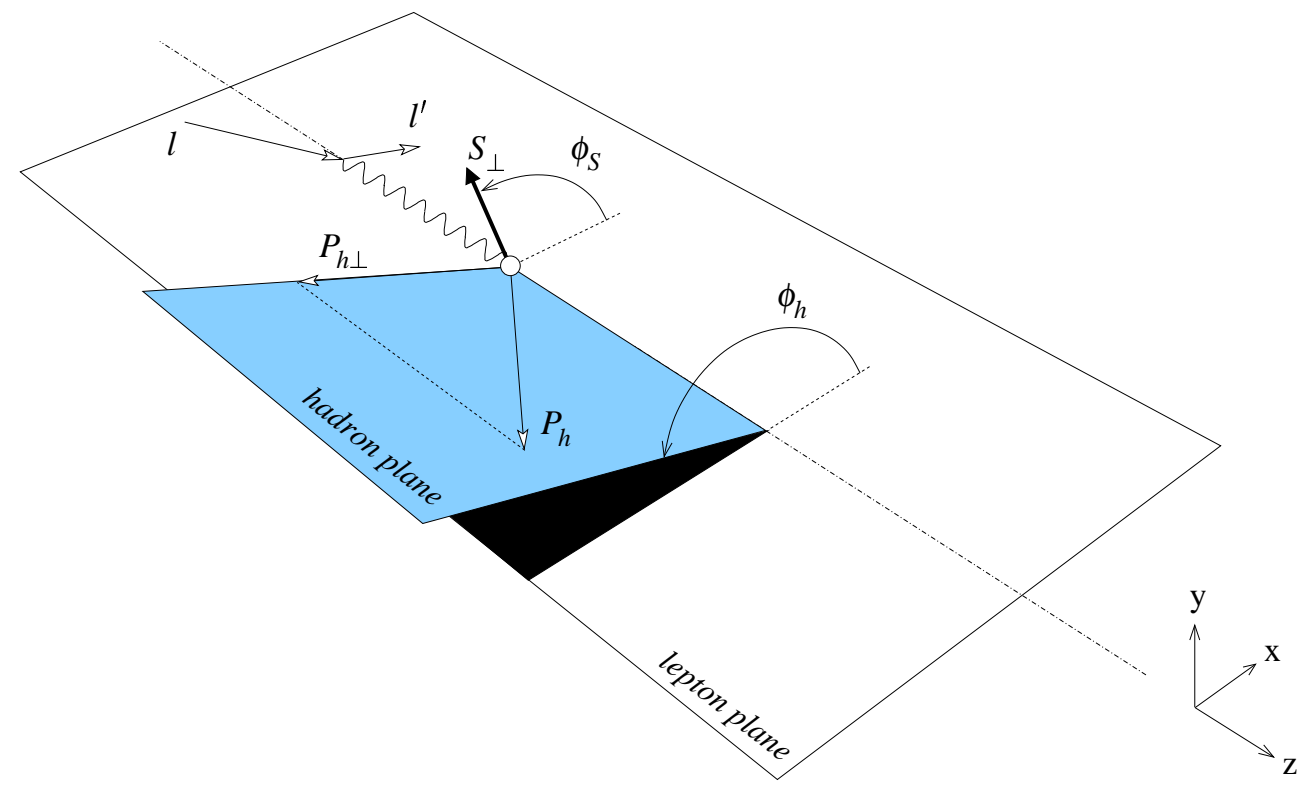

FIG. 1: Definition of azimuthal angles for the process 15 in the target rest frame. $P_{h \perp}$ and $S_{\perp}$ are the components of $P_{h}$ and $S$ transverse to the photon momentum.

Theorists often prefer a coordinate system with the same $x$ axis but with $y$ and $z$ axes opposite to those shown in Fig. 1, so that in the $\gamma^{*} p$ center of mass the target moves in the positive $z$ direction (cf. Sect. I). When working 
in that coordinate system in the context of graphical convention $\AA$ one can conform with the definition of angles recommended here by using the opposite orientation for both $\phi_{h}$ and $\phi_{S}$.

We note that the angles $\phi_{h}$ and $\phi_{S}$ defined here are opposite to those defined in Refs. 1, 2, 3], which must be taken into account when using expressions for azimuthal asymmetries from these papers. ${ }^{9}$

\section{ASYMMETRIES AND AZIMUTHAL MOMENTS}

Longitudinal single-spin asymmetries in lepton-proton scattering should always be defined so that

$$
A\left(\phi_{h}\right) \equiv \frac{d \sigma^{\rightarrow}\left(\phi_{h}\right)-d \sigma^{\leftarrow}\left(\phi_{h}\right)}{d \sigma^{\rightarrow}\left(\phi_{h}\right)+d \sigma^{\leftarrow}\left(\phi_{h}\right)}
$$

where in the case of a beam spin asymmetry $d \sigma \rightarrow$ refers to positive helicity of the lepton. In the case of a target spin asymmetry $d \sigma \rightarrow$ denotes target polarization opposite to the direction either of the lepton beam or of the virtual photon. ${ }^{10}$ Azimuthal moments associated with beam or target spin asymmetries are defined as, e.g.

$$
\left\langle\sin \phi_{h}\right\rangle \equiv \frac{\int d \phi_{h} \sin \phi_{h}\left[d \sigma^{\rightarrow}\left(\phi_{h}\right)-d \sigma^{\leftarrow}\left(\phi_{h}\right)\right]}{\int d \phi_{h}\left[d \sigma^{\rightarrow}\left(\phi_{h}\right)+d \sigma^{\leftarrow}\left(\phi_{h}\right)\right]}
$$

and similarly for $\left\langle\sin 2 \phi_{h}\right\rangle$ etc. As an alternative notation one may use $A^{\sin \phi_{h}}=2\left\langle\sin \phi_{h}\right\rangle{ }^{11}$ If the cross section is of the form

$$
\begin{aligned}
& \frac{d \sigma^{\rightarrow}}{d \phi_{h}}=a_{0}+a_{1} \sin \phi_{h}, \\
& \frac{d \sigma^{\leftarrow}}{d \phi_{h}}=a_{0}-a_{1} \sin \phi_{h}
\end{aligned}
$$

then $A^{\sin \phi_{h}}=a_{1} / a_{0}$ has values between -1 and +1 , as is natural for an asymmetry.

The single spin asymmetry for transverse target polarization can be written as

$$
A\left(\phi_{h}, \phi_{S}\right) \equiv \frac{d \sigma\left(\phi_{h}, \phi_{S}\right)-d \sigma\left(\phi_{h}, \phi_{S}+\pi\right)}{d \sigma\left(\phi_{h}, \phi_{S}\right)+d \sigma\left(\phi_{h}, \phi_{S}+\pi\right)}
$$

and associated azimuthal moments as, e.g.

$$
\left\langle\sin \left(\phi_{h}+\phi_{S}\right)\right\rangle \equiv \frac{\int d \phi_{h} d \phi_{S} \sin \left(\phi_{h}+\phi_{S}\right)\left[d \sigma\left(\phi_{h}, \phi_{S}\right)-d \sigma\left(\phi_{h}, \phi_{S}+\pi\right)\right]}{\int d \phi_{h} d \phi_{S}\left[d \sigma\left(\phi_{h}, \phi_{S}\right)+d \sigma\left(\phi_{h}, \phi_{S}+\pi\right)\right]}
$$

and similarly for $\left\langle\sin \left(\phi_{h}-\phi_{S}\right)\right\rangle$ etc. It should be straightforward to generalize these conventions to the case of double spin asymmetries and of $\left|P_{h \perp}\right|$-weighted asymmetries [2].

\section{Acknowledgments}

We thank the organizers and all the participants of the workshop "Transversity: New Developments in Nucleon Spin Structure" (ECT*, Trento, Italy, 14-18 June 2004). Special thanks are due to M. Anselmino, D. Boer, A. Metz, P.J. Mulders, F. Murgia, F. Pijlman, M. Radici, and G. Schnell for valuable input to the manuscript. This work is part of the EU Integrated Infrastructure Initiative "Study of strongly interacting matter (HadronPhysics)" under contract number RII3-CT-2004-506078.

[1] P. J. Mulders and R. D. Tangerman, Nucl. Phys. B461, 197 (1996), erratum-ibid. B484, 538 (1996), hep-ph/9510301.

\footnotetext{
9 There is an inconsistency in Fig. 1 of Ref. 3] and Fig. 1 of Ref. [2]: according to the formulae given in those papers, the azimuthal angle shown in those figures (which is positive according to graphical convention $\mathrm{A}$ ) is equal to $-\phi$ and not to $\phi$.

10 Note that target polarization opposite to the virtual photon momentum corresponds to positive helicity of the proton in the $\gamma^{*} p$ center of mass.

${ }^{11}$ In the literature sometimes the factor 2 is not included, a choice that we do not recommend.
} 
[2] D. Boer and P. J. Mulders, Phys. Rev. D57, 5780 (1998), hep-ph/9711485.

[3] D. Boer, R. Jakob, and P. J. Mulders, Nucl. Phys. B564, 471 (2000), hep-ph/9907504.

[4] M. Anselmino, M. Boglione, and F. Murgia, Phys. Rev. D60, 054027 (1999), hep-ph/9901442.

[5] M. Anselmino, M. Boglione, and F. Murgia, Phys. Lett. B362, 164 (1995), hep-ph/9503290.

[6] V. Barone, A. Drago, and P. G. Ratcliffe, Phys. Rept. 359, 1 (2002), hep-ph/0104283.

[7] D. W. Sivers, Phys. Rev. D41, 83 (1990).

[8] M. Anselmino, U. D'Alesio, and F. Murgia, Phys. Rev. D67, 074010 (2003), hep-ph/0210371.

[9] M. Boglione and P. J. Mulders, Phys. Rev. D60, 054007 (1999), hep-ph/9903354.

[10] J. C. Collins, Nucl. Phys. B396, 161 (1993), hep-ph/9208213.

[11] M. Anselmino and F. Murgia, Phys. Lett. B483, 74 (2000), hep-ph/0002120.

[12] M. Anselmino, D. Boer, U. D'Alesio, and F. Murgia, Phys. Rev. D65, 114014 (2002), hep-ph/0109186.

[13] J. C. Collins, Phys. Lett. B536, 43 (2002), hep-ph/0204004. 